\title{
Human cervicovaginal mucus contains an activity that hinders HIV-1 movement
}

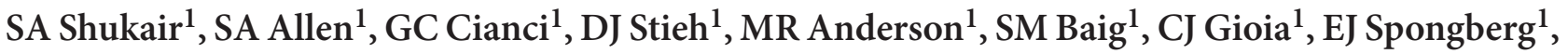 \\ SM Kauffman ${ }^{1}$, MD McRaven ${ }^{1}$, HY Lakougna ${ }^{2}$, C Hammond ${ }^{3}$, PF Kiser ${ }^{2,4}$ and TJ Hope ${ }^{1}$
}

Cervical and vaginal epithelia are primary barriers against HIV type I (HIV-1) entry during male-to-female transmission. Cervical mucus (CM) is produced by the endocervix and forms a layer locally as well as in the vaginal compartment in the form of cervicovaginal mucus (CVM). To study the potential barrier function of each mucus type during HIV-1 transmission, we quantified HIV-1 mobility in CM and CVM ex vivo using fluorescent microscopy. Virions and 200-nm PEGylated beads were digitally tracked and mean-squared displacement was calculated. The mobility of beads increased significantly in CVM compared with CM, consistent with the known decreased mucin concentration of CVM. Unexpectedly, HIV-1 diffusion was significantly hindered in the same CVM samples in which bead diffusion was unhindered. Inhibition of virus transport was envelope-independent. Our results reveal a previously unknown activity in CVM that is capable of impeding HIV-1 mobility to enhance mucosal barrier function.

\section{INTRODUCTION}

To initiate infection in the female reproductive tract, HIV type I (HIV-1) must infect susceptible immune cells protected by either the stratified, squamous epithelium of the vaginal compartment or the simple, columnar epithelium of the cervical canal. ${ }^{1,2}$ In both cases, the virus must first traverse a layer of mucus found covering these surfaces. This mucus layer contains various innate and adaptive immunity components, including physical barrier properties, which may provide important functions during exposure to HIV-1. ${ }^{3-5}$ A more detailed understanding of the natural barrier function provided by mucus lining the cervical canal and the vaginal compartment will assist in finding methods to fortify the innate mechanisms protecting the female reproductive tract.

Mucins form highly viscoelastic biopolymer networks that allow mucus to act as a lubricant and physical barrier for microbes at mucosal interfaces. ${ }^{3}$ Cell-surface mucins (MUC1, MUC4, and MUC16) and gel-forming secreted mucins (MUC5AC, MUC5B, and MUC6) have been imaged at the endocervical epithelium and found within cervical mucus (CM) ${ }^{6,7}$ All mucosal epithelial cells produce and express cellsurface mucin glycoproteins, while goblet cells produce and secrete gel-forming mucins, and both of these mucin types are found in the resulting mucus formed within the endocervix. ${ }^{8}$ $\mathrm{CM}$ migrating through the canal is then expelled through the cervical os largely by gravitational flow into the vaginal compartment, ${ }^{9}$ lined by ectocervical and vaginal epithelia, where it mixes with cervicovaginal fluid. Cervicovaginal fluid includes contributions from vaginal wall transudate, vulvar secretions, exfoliated epithelial cells, and secretions from bacterial flora. ${ }^{10}$ This mixture of CM and cervicovaginal fluid is termed cervicovaginal mucus (CVM), and owing to higher fluid content of $\mathrm{CVM}$, it is likely that the mucin structure and barrier function in CVM differs from that in CM secreted at the endocervix.

Despite the potential importance of mucus during sexual transmission, few studies have probed the interaction of HIV-1 with CM or CVM. One study, involving our group, found that the rate of viral diffusion in mid-cycle CVM is pH-dependent, with virus-like particles being potentially immobilized in CVM with $\mathrm{pH} \sim 4$ and more diffusive when CVM was buffered to $\mathrm{pH}$ $\sim 6$ using sodium hydroxide. In addition, there is one published study of HIV-1 in CM, which found that HIV-1 underwent extremely hindered, anomalous diffusion in undiluted CM collected from the cervical os. ${ }^{11}$ Unfortunately, both of these

\footnotetext{
${ }^{1}$ Department of Cell and Molecular Biology, Northwestern University Feinberg School of Medicine, Chicago, Illinois, USA. ${ }^{2}$ Department of Bioengineering, University of Utah, Salt Lake City, Utah, USA. ${ }^{3}$ Department of Obstetrics and Gynecology, Section in Family Planning and Contraception, Northwestern University Feinberg School of Medicine, Chicago, Illinois, USA. ${ }^{2}$ Department of Pharmaceutics and Pharmaceutical Chemistry, University of Utah, Salt Lake City, Utah, USA. Correspondence: TJ Hope (thope@northwestern.edu) 

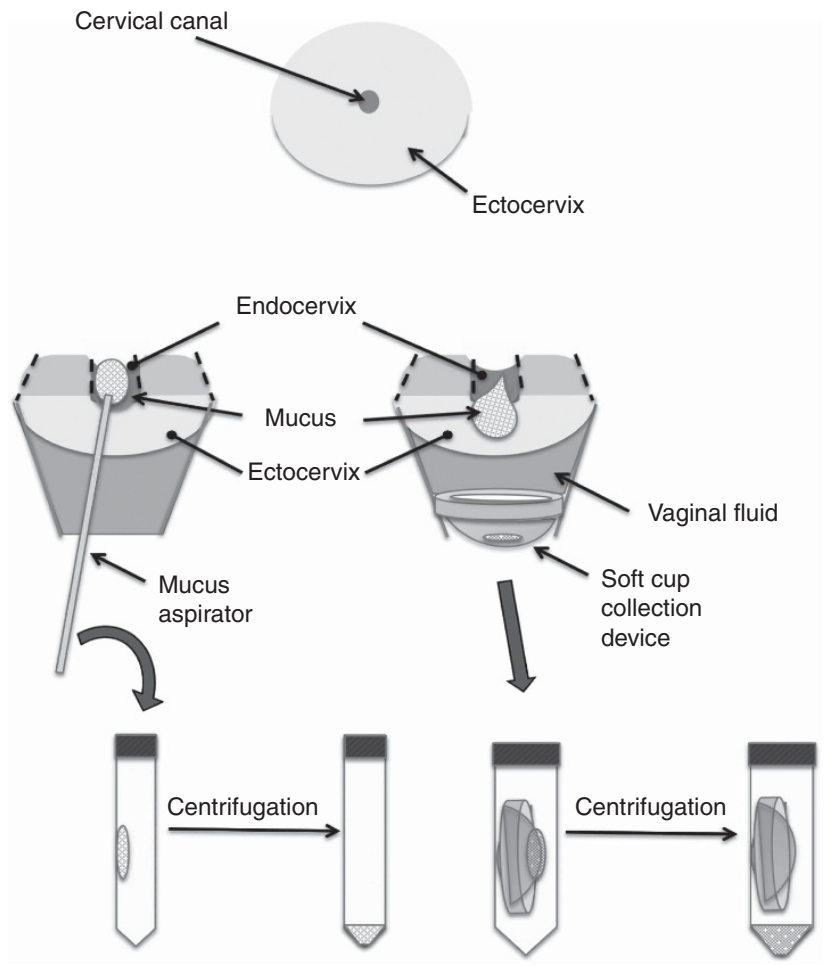

Figure 1 Institutional Review Board-approved collection of cervical mucus (CM) and cervicovaginal mucus (CVM) samples for experimentation. $\mathrm{CM}$ is produced and secreted by the endocervical epithelium and flows into the vaginal vault, where it may mix with vaginal fluid. CM (left) was aspirated directly from the cervical canal at the os during routine gynecological exams and transferred to a $15-\mathrm{ml}$ conical tube for centrifugation. CVM (right) was collected from donors via a selfsampling method involving a commercially available collection cup and transferred to a 50-ml conical tube for centrifugation. Experiments in both mucus types were performed in the same manner.

studies focused on small donor populations $(n<10)$, neglecting the contribution of inter-donor variability. ${ }^{12-14}$ In addition, it is unknown how viral surface proteins affect the ability of HIV-1 to diffuse in CM. For instance, while less than 20 trimers of viral envelope glycoproteins are incorporated into each wild-type (WT) budding virion, Env could interact with factors in CM to impede or enhance the diffusion of HIV- $1^{15}$. Another component of the transmission environment that may affect viral movement in CM is the presence of seminal plasma (SP). It has been shown that the volume of human ejaculate ranges from $2-5 \mathrm{ml},{ }^{16}$ having the ability to dilute CM with seminal fluid, with highest dilution in vaginal epithelial regions where mucus coating is not as thick. ${ }^{9}$

Here, we examined the diffusion of WT and Env-deleted ( $\Delta$ Env) HIV-1 in $95 \mathrm{CM}$ and $22 \mathrm{CVM}$ samples. We also included an additional control of 200 -nm polyethylene glycol (PEG)-modified polystyrene beads (beads) that appear to weakly interact with mucins. ${ }^{17} \mathrm{CM}$ and CVM were collected via distinct methods (Figure 1), and each of these two compartments has distinct attributes. We found that while the diffusion of beads was greater in CVM samples relative to CM

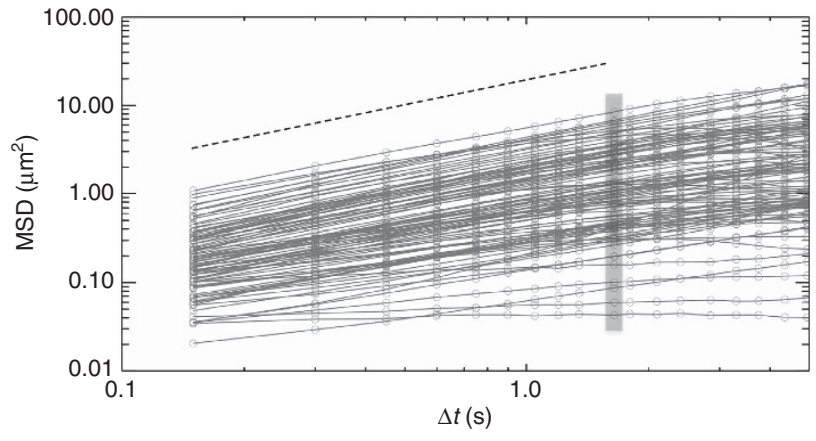

Figure 2 Compilation of mean-squared displacement (MSD) and $\alpha$ data for particles in mucus. MSD values for wild-type (WT) and $\Delta$ Env virus and beads were plotted against timescale $(\Delta t)$ on a log-log plot. This is a representative graph with data for WT HIV-1 in cervical mucus (CM). Each red curve is the time-averaged ensemble MSD for all particles in an individual CM sample ( $n=95$ donors), in which $\geqslant 100$ particles were tracked. We used MSD values over a timescale of $1.5 \mathrm{~s}$ (highlighted) for comparisons between particle types and mucus types. In addition, power law exponents $(\alpha)$ were calculated for each donor by fitting a line to each curve up to a $\Delta t$ of $1.5 \mathrm{~s}$. The dashed line is a reference for $\alpha=1$.

samples, the diffusion of HIV-1 was slower in CVM compared with CM. This slower diffusion of virus in CVM compared with $\mathrm{CM}$ over the same timescale was not influenced by the presence of the HIV-1 envelope glycoproteins, as both WT and $\triangle E n v$ viral particles diffused significantly less in CVM compared with the beads. In addition, viral mobility in CVM correlated positively with $\mathrm{pH}$, while bead mobility did not. In CM, diffusion of both the beads and HIV-1 particles was hindered as evidenced by $\alpha$ values; however, dilution of CM with either SP or phosphate-buffered saline (PBS) increased the mobility of all particles. Our results demonstrate that CM and CVM are both capable of impeding HIV-1 mobility, and suggest that the mechanism of viral inhibition by CVM involves specific interactions of a mucus component with the membrane surface of viruses.

\section{RESULTS}

Identification of an activity in CVM that hinders the diffusion of HIV-1

To evaluate differences in diffusion of WT HIV-1, $\Delta$ Env HIV-1, and beads in CM compared with CVM, we used the conventional measure of time- and ensemble-averaged mean-squared displacement $(\mathrm{MSD})^{11}$. Ninety-five CM and $22 \mathrm{CVM}$ samples from distinct donors were examined. In order to simplify the comparison of particle movement between donors and mucus types (Figure 2), we chose to focus on MSD values over a timescale $(\Delta t)$ of 1.5 s, i.e., 10 frames (highlighted in Figure 2). This $\Delta t$ included data representing both fast- and slow-moving particles, thereby removing bias from our analysis. Over a $\Delta t$ of $1.5 \mathrm{~s}, \Delta$ Env virus had a greater MSD $\left(1.3 \mu \mathrm{m}^{2}\right)$ than WT virus $\left(1.0 \mu \mathrm{m}^{2}, P<0.05\right)$ and beads $\left(1.1 \mu \mathrm{m}^{2}, P<0.05\right)$ in $\mathrm{CM}$ (Figure 3a, Table 1). In contrast, the MSD values for both virion types in CVM over $1.5 \mathrm{~s}$ timescales were similar, with 

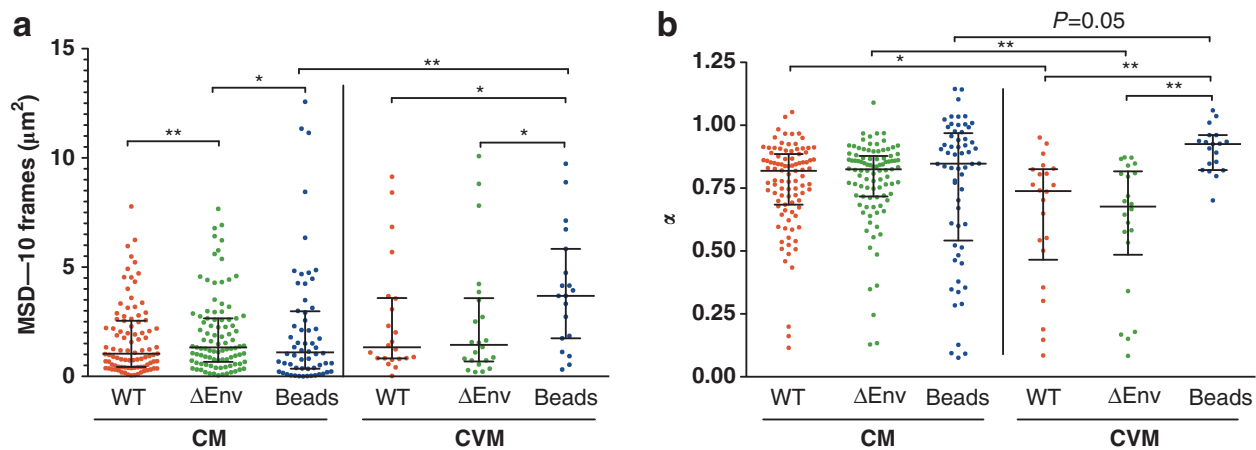

Figure 3 Diffusion of fluorescently labeled particles in cervical mucus (CM) and cervicovaginal mucus (CVM). (a) Mean-squared displacement (MSD) values for wild-type (WT) HIV-1, $\Delta$ Env HIV-1, and beads over a $\Delta t$ of 10 frames were compared in CM and CVM, with each point representing the MSD value of one donor. (b) Fitting a line to each ensemble MSD curve up to a $\Delta t$ of $1.5 \mathrm{~s}$ yielded $\alpha$ values for all particle types in CM and CVM. Wilcoxon signed-rank tests were run on paired particle types in samples from the same donor (WT vs. $\Delta$ Env, WT vs. beads, etc.), while Wilcoxon ranked sum tests were run to compare movement of the same particle type in different mucus types (CM vs. CVM). Median values with interquartile ranges are shown for all donors. ${ }^{*} 0.01>P<0.05,{ }^{* *} P<0.01$.

\begin{tabular}{|c|c|c|c|}
\hline Particle type & $\begin{array}{c}M S D\left(\mu m^{2}\right) \\
\text { median (range) }\end{array}$ & $\alpha$, Median (range) & $\mathrm{n}$ \\
\hline \multicolumn{4}{|l|}{$C M$} \\
\hline WT & $1.0(0.044-7.8)$ & $0.82(0.12-1.1)$ & 95 \\
\hline$\Delta$ Env & $1.3(0.044-7.7)$ & $0.83(0.13-1.1)$ & 95 \\
\hline Beads & $1.1(0.0039-13)$ & $0.85(0.076-1.1)$ & 60 \\
\hline
\end{tabular}

\begin{tabular}{lccc} 
CVM & & & \\
WT & $1.3(0.021-9.1)$ & $0.74(0.085-0.95)$ & 22 \\
$\Delta$ Env & $1.4(0.20-10)$ & $0.68(0.083-0.87)$ & 22 \\
Beads & $3.7(0.32-9.7)$ & $0.93(0.70-1.1)$ & 19 \\
\hline
\end{tabular}

Abbreviations: CM, cervical mucus; CVM, cervicovaginal mucus; MSD, meansquared displacement; WT, wild-type.

medians of $1.3 \mu \mathrm{m}^{2}$ and $1.4 \mu \mathrm{m}^{2}$ for WT and $\Delta \mathrm{Env}$, respectively. The MSD of the beads, however, increased from $1.1 \mu \mathrm{m}^{2}$ to $3.7 \mu \mathrm{m}^{2}$ in CVM $(P<0.01)$, which was significantly higher than both WT $(P<0.05)$ and $\Delta$ Env HIV-1 $(P<0.05)$ (Figure 3a, Table 1). The relative mobility of the viral particles and beads was quite different in CM vs. CVM. Unlike the MSD of the viral particles, which only increased by $0.3 \mu \mathrm{m}^{2}$ in CVM $(P>0.05$, not significant), the MSD of the beads increased by $2.6 \mu \mathrm{m}^{2}$ (3.5-fold) in the CVM $(P<0.01)$. These results reveal that the mobility of WT and $\triangle$ Env HIV-1 is hindered in CVM, while bead mobility is not.

While examining MSD values at a single $\Delta t$ simplifies comparisons, considering MSD values over a range of timescales allows for a more proper characterization of the microscopic motion of particles. We therefore also adopted a simple approach of fitting the MSD data to a power law:

$$
M S D=C \Delta t^{\alpha}
$$

For free diffusion, the power law exponent, $\alpha$, is equal to one (Figure 2, dashed line). When $\alpha$ is less than one, the motion is hindered or sub-diffusive, indicating that the particles are experiencing a retarding interaction with their environment. ${ }^{17}$ To obtain $\alpha$ values for each particle type, we fit a line through the MSD data up to a $\Delta t$ of $1.5 \mathrm{~s}$. Up to this timescale, we found that $\alpha$ values for $\mathrm{WT}$ and $\Delta \mathrm{Env}$ virus were significantly lower in CVM than in CM. The median $\alpha$ value for WT decreased from 0.82 to $0.74(P<0.05)$ while the median $\alpha$ value for $\Delta$ Env virus decreased from 0.83 to $0.68(P<0.01)$ in CVM relative to $\mathrm{CM}$, respectively. (Figure 3b, Table $\mathbf{1}$ ). In contrast, the $\alpha$ values for the beads increased in CVM relative to CM. The median $\alpha$ value for the beads in $\mathrm{CM}$ was 0.85 , while the median $\alpha$ value for beads in CVM was $0.93(P=0.06)$, indicating that the mobility of the beads was unhindered in CVM (Figure 3b, Table 1). The observation that the $\alpha$ values for beads increase in CVM relative to $\mathrm{CM}$ while the $\alpha$ values for the viral particles decrease further supports the model that mobility of WT and $\Delta$ Env HIV- 1 is hindered in CVM by an interaction. In addition, we found that the coefficient of variation (CV) for bead $\alpha$ values in CVM was extremely low $(\mathrm{CV}=9.87 \%)$ compared with $\mathrm{WT}(\mathrm{CV}=41.50 \%)$ and $\Delta \mathrm{Env}(\mathrm{CV}=42.62 \%) \mathrm{HIV}-1$. In contrast, the $\mathrm{CV}$ for $\alpha$ values was much higher for beads in $\mathrm{CM}(\mathrm{CV}=38.05 \%)$, where beads exhibited more variable movement than both $\mathrm{WT}(\mathrm{CV}=22.80 \%)$ and $\triangle \operatorname{Env}(C V=21.69 \%)$ viral particles.

\section{Mobility of HIV-1 in CVM, but not in CM, positively correlates with $\mathrm{pH}$ of the mucus sample}

To further assess differences in mobility between the particles in CM and CVM, the effect of $\mathrm{pH}$ on MSD and $\alpha$ was examined in 72 samples for which mucus sample volume was high enough to obtain a reliable $\mathrm{pH}$ measurement. The $\mathrm{CM}$ and CVM samples ranged in $\mathrm{pH}$ from 4.4-9.4 and 4.0-8.9, respectively, with both mucus types demonstrating a normal distribution $(P>0.10)$ as assessed using the D'Agostino-Pearson omnibus normality test (Figure 4). The $52 \mathrm{CM}$ samples exhibited an average $\mathrm{pH}$ of $7.2 \pm 1.0$, while the average $\mathrm{pH}$ of the $20 \mathrm{CVM}$ samples was 
5.8 \pm 1.4. We determined the extent of correlation between mucus $\mathrm{pH}$ and particle diffusion by plotting the MSD values as a function of $\mathrm{pH}$ and calculating the Spearman correlation coefficient $\left(r_{\mathrm{s}}\right)$ for each particle type in CM and CVM. MSD values and $\mathrm{pH}$ correlated significantly in a positive manner for WT $\left(r_{\mathrm{s}}=0.60\right.$, $P<0.01)$ and $\Delta$ Env $\left(r_{\mathrm{s}}=0.61, P<0.01\right) \mathrm{HIV}-1$ particles in $\mathrm{CVM}$ samples (Figure 5b), with decreasing MSD values at decreasing $\mathrm{pH}$. This correlation, however, between MSD values of either particle type and $\mathrm{pH}$ was not significant in CM (Figure 5a). The MSD values for beads did not significantly correlate with $\mathrm{pH}$ in either CM or CVM (Figure 5). In addition, when we examined the correlation between $\alpha$ and $\mathrm{pH}$ in our mucus samples, there was also a statistically significant positive correlation $\left(r_{\mathrm{s}}=0.44\right.$, $P<0.05$ ) for WT HIV-1 in CVM (Figure 6b). No significant correlation was found between $\alpha$ and $\mathrm{pH}$ for WT HIV-1 in CM or $\Delta \mathrm{Env} H \mathrm{HV}-1$ and beads in either mucus type (Figure 6).

\section{Dilution of CM with SP increases MSD of particles}

During male-to-female sexual transmission, virus encounters susceptible tissues in the female genital tract via a semen

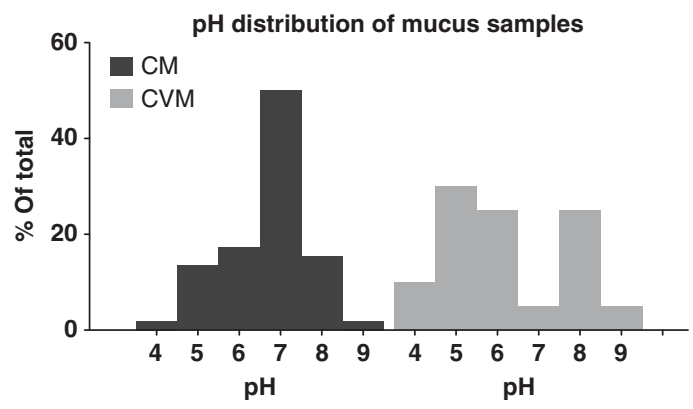

Figure 4 pH distribution among cervical mucus (CM) and cervicovaginal mucus (CVM) samples. $\mathrm{pH}$ values of mucus samples were binned in increments of $1.0 \mathrm{pH}$ unit, and the $y$ axis quantifies these binned values as a percentage of the total number of samples of respective mucus type. The total number of samples with $\mathrm{pH}$ data for $\mathrm{CM}$ was 52 and for CVM was 20. vehicle. To examine the potential impact of semen on diffusion of HIV-1 in CM, we imaged virus and beads in 10 samples before and after mixing with an equal volume of SP utilized here as a surrogate for semen. We removed cells via centrifugation from a commercially available pooled semen sample to generate SP. CM samples were divided into three aliquots, and viral particles and beads were added directly to one aliquot for imaging. We mixed a second aliquot of each CM sample with an equal volume of SP before particles were added for imaging, and further mixed a third aliquot with an equal volume of PBS as a control to differentiate between the general effects of dilution and effects that may be SP-specific. In these samples, both the PBS and SP significantly increased the MSD and $\alpha$ values of WT viral particles in CM (Figure 7a, Table 2). The median MSD value over a 1.5-s timescale for WT HIV-1 in those 10 donors rose from $0.38 \mu \mathrm{m}^{2}$ to $1.1 \mu \mathrm{m}^{2}$ when that $\mathrm{CM}$ was mixed with either PBS $(P<0.05)$ or SP $(P<0.01)$. However, only the addition of SP significantly raised the median MSD of beads in CM from 0.25 to $1.2 \mu \mathrm{m}^{2}$ and of $\Delta \mathrm{Env} H I V-1$ in $\mathrm{CM}$ from 0.94 and $0.97 \mu \mathrm{m}^{2}(P<0.01)$ (Figure 7a, Table 2). The $\alpha$ values were consistent with the differences in MSD (Figure 7b, Table 2).

In addition to the CM samples discussed thus far, there were $41 \mathrm{CM}$ samples with either low starting volume or extremely elastic characteristics. In order to allow for experimentation with these samples, they were premixed with an equal volume of SP before imaging. As the donors in the $\mathrm{CM}+\mathrm{SP}$ group and the $\mathrm{CM}$ alone group are unrelated, this was a noncorrelative assessment of particle diffusion in CM with or without SP. In SP alone, viral particles and beads diffused unhindered as evidenced by median $\alpha$ values of $\sim 1$ (Supplementary Figure 1 and Supplementary Table S1). This was significantly higher than particle diffusion in an equal mixture of SP and CM $(P<0.01)$, where the MSD and $\alpha$ values for all particles were significantly lower than in SP alone (Supplementary Figure 1 and Supplementary Table S1). Unlike virus, the median MSD value for beads in
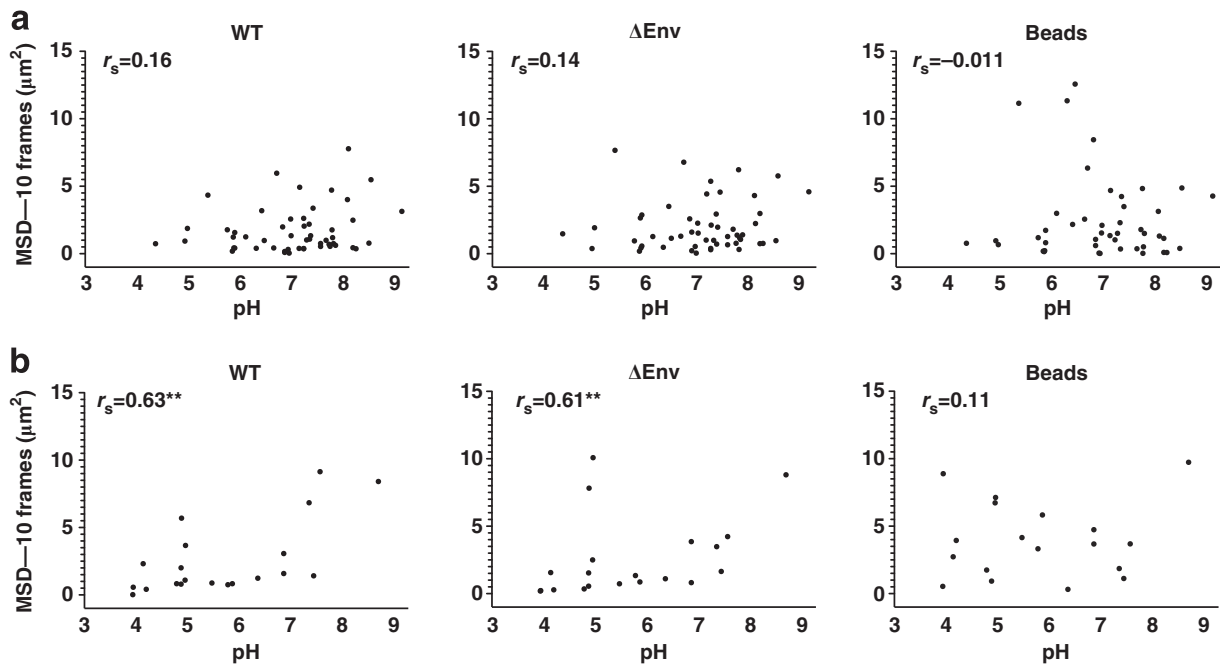

Figure 5 Correlation of particle mean-squared displacement (MSD) values with pH in cervical mucus (CM) and cervicovaginal mucus (CVM). MSD values over a $\Delta t$ of $1.5 \mathrm{~s}$ were obtained and plotted as a function of $\mathrm{pH}$ of the (a) $\mathrm{CM}$ or (b) CVM sample to calculate Spearman correlation coefficients. ${ }^{* \star} P<0.01$. 

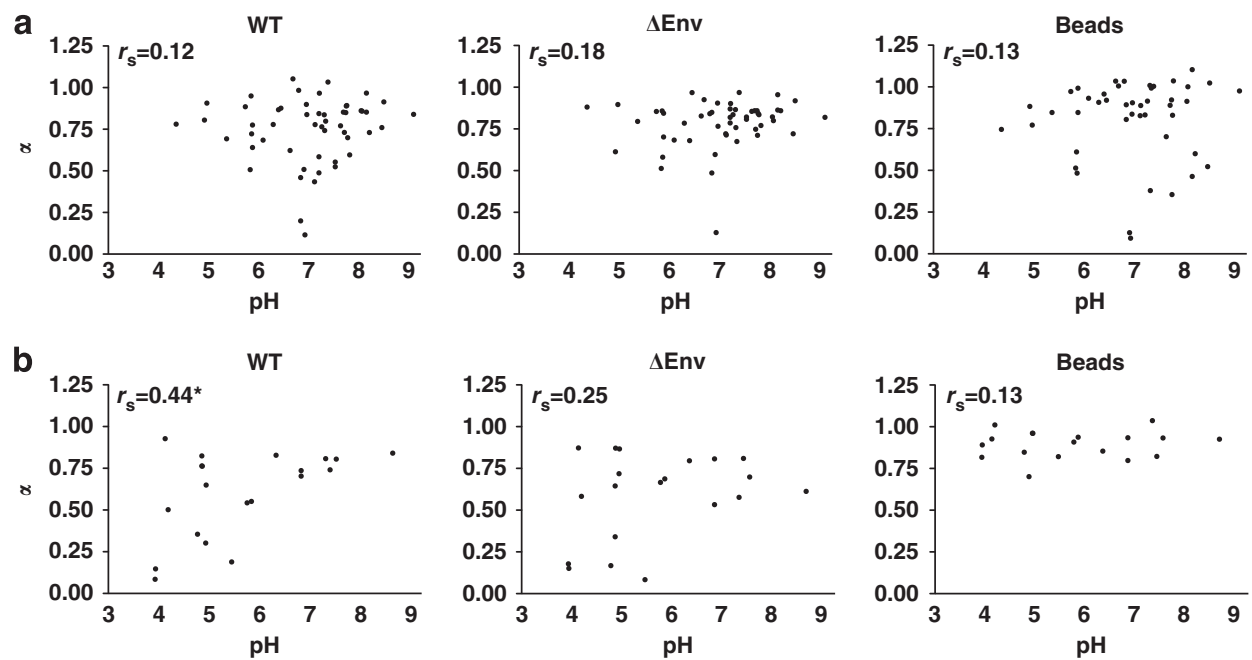

Figure 6 Correlation of $\alpha$ with pH in cervical mucus (CM) and cervicovaginal mucus (CVM). Power law exponents for each donor were obtained and plotted as a function of sample pH in (a) CM and (b) CVM. Spearman correlation coefficients $\left(r_{\mathrm{s}}\right)$ are shown along with significant $P$-values. ${ }^{\star} 0.01>P<0.05$. WT, wild type.
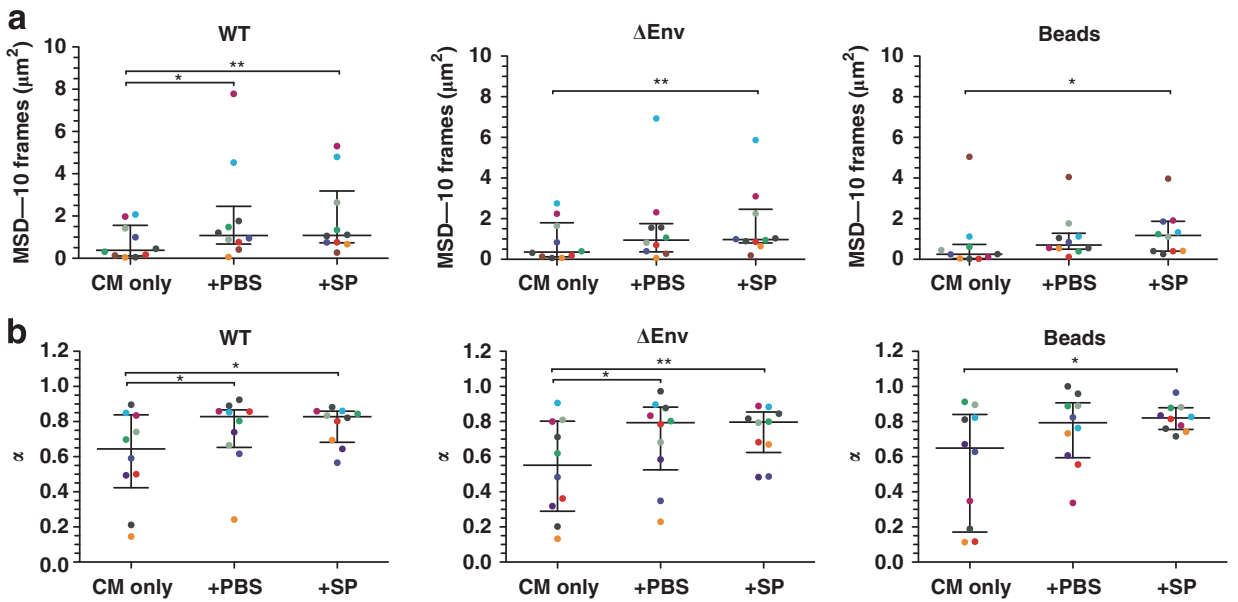

Figure 7 Comparison of particle diffusion in native cervical mucus (CM) and CM diluted with seminal plasma (SP). In CM from 10 donors, the (a) mean-squared displacement (MSD) and (b) $\alpha$ values for particles were obtained in CM alone (CM only) or in CM mixed with phosphate-buffered saline (PBS) (+PBS) or SP (+SP). Each color represents a different donor. Wilcoxon signed-rank tests were used for paired particle types in samples from the same donor, while Wilcoxon ranked sum tests were run to compare movement between the same type of particle in either the presence or absence of SP. Bars represent median values with interquartile ranges shown. ${ }^{\star} 0.01>P<0.05,{ }^{\star \star} P<0.01$.

CM with SP was not significantly different from beads in CM alone (Supplementary Figure 1, Table S1).

\section{DISCUSSION}

In summary, our findings reveal an interaction in CVM that impedes HIV-1 movement but not the movement of 200-nm PEGylated beads. The significantly higher MSD values for beads in CVM when compared with WT and $\triangle$ Env viral particles, despite the slightly larger diameter of the beads, indicates that both virion types are interacting with components within CVM to hinder virus transport. As the viral membrane present on both WT and $\Delta$ Env virions contains multiple host-cell-derived proteins and lipids, the decrease in diffusion of viral particles compared with beads is likely due to interactions between the milleiu of cellular lipids and proteins captured in viral particles and a protein(s) in CVM. ${ }^{18-20}$ This Env independence suggests that the impeding mechanism in CVM may have evolved as a defense against enveloped viruses in general.

In contrast, $\mathrm{CM}$ samples consistently hindered the movement of both viral particles and beads. WT virus diffused less in $\mathrm{CM}$ than $\Delta \mathrm{Env}$ virus; however, while statistically significant, the biological consequences of a small $\left(0.2-0.3 \mu \mathrm{m}^{2}\right)$ difference in MSD is unclear. The difference could be a result of charge interactions between ENV glycan groups and those that decorate mucins in CM. The small difference in MSD between virion types observed in $\mathrm{CM}$ is absent in $\mathrm{CVM}$, indicating that either the ENV-dependent interactions that were slowing WT virus in CM are no longer occurring or that the effects of 
Table 2 Effects of dilution on particle movement in $\mathrm{CM}$ from 10 donors

\begin{tabular}{|c|c|c|}
\hline & $\begin{array}{c}M S D\left(\mu m^{2}\right), \\
\text { median (range) }\end{array}$ & $\alpha$, Median (range) \\
\hline \multicolumn{3}{|l|}{$W T$} \\
\hline CM only & $0.38(0.044-2.1)$ & $0.64(0.15-0.90)$ \\
\hline $\mathrm{CM}+\mathrm{PBS}$ & $1.1(0.055-7.8)$ & $0.83(0.24-0.92)$ \\
\hline $\mathrm{CM}+\mathrm{SP}$ & $1.1(0.27-5.3)$ & $0.83(0.57-0.88)$ \\
\hline \multicolumn{3}{|l|}{$\Delta E n v$} \\
\hline CM only & $0.36(0.064-2.7)$ & $0.55(0.13-0.91)$ \\
\hline $\mathrm{CM}+\mathrm{PBS}$ & $0.94(0.064-6.9)$ & $0.79(0.23-0.97)$ \\
\hline $\mathrm{CM}+\mathrm{SP}$ & $0.97(0.19-5.9)$ & $0.80(0.48-0.89)$ \\
\hline \multicolumn{3}{|l|}{ Beads } \\
\hline CM only & $0.25(0.020-5.0)$ & $0.65(0.11-0.91)$ \\
\hline $\mathrm{CM}+\mathrm{PBS}$ & $0.70(0.12-4.0)$ & $0.79(0.34-1.0)$ \\
\hline $\mathrm{CM}+\mathrm{SP}$ & $1.2(0.25-4.0)$ & $0.82(0.72-0.96)$ \\
\hline
\end{tabular}

Abbreviations: CM, cervical mucus; MSD, mean-squared displacement; PBS, phosphate-buffered saline; SP, seminal plasma; WT, wild-type.

ENV-independent interactions on both virion types in CVM overpower them. In agreement with the MSD values, $\alpha$ values suggest that the virions undergo retarding interactions with CVM that the beads do not, and that these interactions are ENVindependent. Various cell-derived proteins and/or glycans may have a role in hindering the mobility of HIV-1 in mucus, and further experiments could delineate which, if any, of these are involved.

Physical hindrance in CM by a dense mucin network is likely responsible for the lower MSD values for beads in CM compared with CVM. Nevertheless, a change in the surface properties of the zwitterionic beads in some CM samples may have led to interactions with a CM component, thereby slowing the diffusion of the beads (Supplementary Figure 2). The low level of variability of $\alpha$ values for beads between samples in CVM was in contrast to the variation observed for bead $\alpha$ values in $\mathrm{CM}$, where bead diffusion was more variable than bead diffusion in CVM and viral diffusion in both types of mucus. The wide range of diffusive behavior experienced by the beads in CM compared with CVM may reflect sampling at many points in the menstrual cycle, as we collected all CVM samples at the self-reported midcycle phase, while menstrual cycle data for CM samples in our study were not available at the time of collection. As it has been demonstrated in CM that the water content, mucin expression levels, and glycosylation vary throughout the cycle, ${ }^{8,12,13}$ further studies should determine whether behaviors observed correlate with specific points within the menstrual cycle.

Another possible explanation for the variability we observed in bead diffusion in CM is hormonal contraceptive use. That 20 of the 22 CVM donors were naturally cycling may account for the low variability in bead behavior between CVM samples. While no information was available regarding contraceptive use for CM donors, ongoing studies will account for this variable as it has recently been shown that initiation of a common oral contraceptive leads to differences in mucus content. Further still, there are genotypic differences between individuals that affect the number and type of carbohydrate residues on mucins. ${ }^{3,21-24}$ These differences may affect the viscoelastic properties of mucus as well as the possible interactions between mucins and enveloped viruses, such as HIV-1. Studying matched CM and CVM samples from the same donors would remove inter-donor mucin heterogeneity as a cause of differences in bead and HIV-1 diffusion in the two mucus types.

The significant correlation we observed in CVM between $\mathrm{pH}$ and MSD for viral particles indicates that the interaction occurring between CVM and HIV-1 is favored at low $\mathrm{pH}$. This observation, coupled with the lack of correlation between $\mathrm{pH}$ and MSD for beads in CVM, suggests that changes in $\mathrm{pH}$ affect a direct interaction between a component of CVM and the viral membrane. Had the difference in behavior indicated a major change in mucus structure, the beads would be hindered as well at low $\mathrm{pH}$. Although this is the case, interestingly, CM and CVM samples covered a broad range of $\mathrm{pH}$ values, with some slight skewing toward $\mathrm{pH} 7$ for $\mathrm{CM}$ and $\mathrm{pH} 4$ for CVM.

The ability of CM to potently inhibit the mobility of HIV-1 at the endocervical surface has important implications for the mechanism(s) of male-to-female transmission. Mucus has long been overlooked as a barrier in studies that implicate the endocervix as a likely portal of entry for the virus during maleto-female HIV-1 transmission. Two-dimensional tracking methods limit analyses to the study of short-range movement. Thus extrapolation must be used to assess the movement of HIV-1 in $\mathrm{CM}$ over the longer timescales relevant to sexual transmission. As $\mathrm{CM}$ is viscoelastic, with an elastic modulus that dominates over medium timescales, extrapolating from short timescale data captured in this study is likely an overestimation of the potential displacement. Using equation (1), we calculated that HIV-1 could diffuse $\sim 2500 \mu^{2}$ in CM over a 6-h interval, which is equivalent to a 50 - $\mu \mathrm{m}$-thick layer. The estimated half-life of free virions in plasma is $\sim 6 \mathrm{~h},{ }^{25}$ and this half-life is likely much shorter in the presence of antimicrobial compounds (i.e., beta defensins $^{26,27}$ and complement proteins ${ }^{28}$ ) in CM. Therefore, by slowing the ability of HIV-1 to reach the surface, the CM barrier also increases the time available for innate immune factors to inactivate virus. In addition, the mucus is moving outward allowing for clearance of an entire volume of mucus from the cervical canal in $\sim 24 \mathrm{~h} .{ }^{29,30}$ Therefore, the dynamic barrier function of CM likely provides a potent barrier to protect the columnar epithelium of the endocervix from interaction with HIV-1 during sexual transmission.

Furthermore, experiments involving the mixing of native $\mathrm{CM}$ with SP suggest that the viscoelastic properties of $\mathrm{CM}$ are capable of influencing this mixture, with an equal amount of CM added to SP significantly hindering the diffusion of virus compared with SP alone. Further studies are needed to delineate how other fluids present during intercourse (i.e., fluid produced by the Bartholin's glands) may affect the mixture; however, it seems clear that mucus provides some additional protection to 
the epithelial barriers exposed during sexual transmission. In addition, the SP used in this study was a pooled specimen, and SP from individuals may exhibit greater or lesser dominance over the mixture consistency than the pooled specimen.

In conclusion, our data reveal that native $\mathrm{CM}$ and $\mathrm{CVM}$ have the ability to impede the diffusion of HIV-1, apparently via distinct mechanisms. Consistent with a potential protective role for mucus, it is known that bacterial vaginosis can disrupt mucus and is associated with increased HIV-1 acquisition. ${ }^{31-33}$ Decreased mucus function would allow more virions to reach the underlying epithelial barriers and potentially initiate transmission. Therefore, the development of mucosal vaccines or microbicides to prevent HIV-1 transmission should consider the contribution of mucus function. Most importantly, such interventions should attempt to enhance rather than disrupt this innate mechanism of protection from HIV-1 transmission.

\section{METHODS}

Collection of CVM and CM. CVM and CM samples were obtained from donors after written informed consent, under a protocol approved by the Institutional Review Board at Northwestern University. All CVM samples were obtained using a self-sampling method at selfreported mid-cycle during the menstrual cycle from premenopausal donors. ${ }^{34}$ Twenty of the 22 CVM donors were naturally cycling and the remaining two were taking oral contraceptives. Briefly, the Instead SoftCup (EvoFem, San Diego, CA) was inserted into the vagina for a minimum of $3 \mathrm{~h}$ by each donor, removed, and placed into a $50-\mathrm{ml}$ centrifuge tube (Figure 1). CM samples were aspirated with a mucus collection device, Mucat (Sepal Reproductive Devices, Boston, MA) directly from the cervical os of premenopausal women undergoing annual gynecological examinations. The CM samples were stored in 15-ml conical tubes (Figure 1). All CM and CVM samples were centrifuged at $780 \times g$ for $10 \mathrm{~min}$, and the clear top layer was collected using a positive displacement pipette for $\mathrm{pH}$ determination. The $\mathrm{pH}$ of all mucus samples was measured using a small-volume $\mathrm{pH}$ electrode (Metrohm, Riverview, FL), and samples were kept at $4{ }^{\circ} \mathrm{C}$ until their use within $48 \mathrm{~h}$ of collection. Pooled human SP used in experiments was purchased from Lee Biosolutions (St. Louis, MO) and stored at $-80^{\circ} \mathrm{C}$ until used.

Cell culture. Unless otherwise indicated, all cells were cultured at $37^{\circ} \mathrm{C}$ under 5\% CO2 in Dulbecco's Modified Eagle Medium w/high glucose, $4.0 \mathrm{~mm}$ L-glutamine, $4500 \mathrm{mg} \mathrm{L1}$ glucose, and no sodium pyruvate, with $10 \%$ fetal bovine serum, and $50 \mathrm{mg} \mathrm{ml}^{-1}$ penicillin/streptomycin (Gibco, Invitrogen, Carlsbad, CA).

Preparation and characterization of fluorescent HIV-1 particles. Virus was produced by polyethylenimine (Polysciences, Warrington, PA) co-transfection of a human embryonic kidney cell line (293T) with various proviral and fluorescent protein constructs. In the case of HIV-1 (WT), a proviral plasmid encoding the R9 strain of virus with BaL envelope was transfected into $293 \mathrm{~T}$ cells, along with a plasmid encoding a Gag protein with a C-terminal mCherry or eGFP Fusion (Gag-cherry or Gag-GFP, respectively). For the viral particles lacking Env glycoproteins $(\Delta \mathrm{Env})$, proviral plasmids containing the R7 genome, with Env deleted, were co-transfected with the Gag-GFP or Gag-Cherry constructs. Cells were washed with $1 \times$ PBS 16-24h following transfection, and fresh Dulbecco's Modified Eagle Medium was added for another 16-24h period, after which supernatant containing labeled virus was collected and concentrated 10 -fold by ultracentrifugatio for $1 \mathrm{~h}$ at 32,000 r.p.m. through a $30 \%$ sucrose cushion.

The $\mathrm{p} 24$ content of the virus preparations before and after ultracentrifugation were determined using a commercially available p24
ELISA kit (PerkinElmer, Waltham, MA). Only viral preparations with a p24 concentration between 800 and $2000 \mathrm{ng} \mathrm{ml}^{-1}$ based on this ELISA assay were used. In addition, all virus preparations were assayed for infectivity using GHOST or TZM- $\beta 1$ indicator cells. Finally, fluorescent protein incorporation was assessed by measuring co-localization of HIV-1 capsid (CA) protein and the given Gag fluorescent protein (Gag-GFP or Gag-cherry) by staining of virions bound to coverslips with a mAb against CA (AG3.0). Only viral preparations that contained at least $85 \%$ incorporation of the fluorescent $\mathrm{Gag}$ proteins were used in experiments.

Synthesis and characterization of PEGylated beads. Materials: MES sodium salt, $N$-(3-dimethylaminopropyl)- $N^{\prime}$-ethylcarbodiimide hydrochloride (EDAC), and $\alpha, \omega$-diamino(PEG) (2000 Da) were purchased from Sigma-Aldrich (St. Louis, MO). Carboxylated nanoparticles $d=200 \mathrm{~nm}\left(\mathrm{EX} 660 \mathrm{~nm}, \mathrm{EM} 690 \mathrm{~nm}, 81 \mu \mathrm{mol} \mathrm{CO}_{2} \mathrm{H} / \mathrm{g}\right)$ were purchased from Invitrogen. Pluronic F-127 was purchased from Spectrum Chemicals (Gardena, CA).

Conjugation of $\alpha, \omega$-diamino(PEG) to the nanoparticles: To $4 \mathrm{ml}$ of an aqueous suspension of $5 \mathrm{mg} \mathrm{ml}^{-1}$ carboxylated nanoparticles $(1.6 \mu \mathrm{mol}$ of $\left.\mathrm{RCO}_{2} \mathrm{H}=1 \mathrm{eq}\right), 120 \mu \mathrm{l}$ of MES buffer ( $\mathrm{pH} 6.5$ ) was added. Next, $24 \mathrm{mg}$ of $\alpha, \omega$-diamino(PEG) $\left(2,000 \mathrm{~g} \mathrm{~mol}^{-1}, 12 \mu \mathrm{mol}, 7.5 \mathrm{eq}\right)$ was added as a solid and dissolved. Then Pluronic F-127 $\left(20 \mathrm{mg} \mathrm{ml}^{-1}\right)$ was added to a concentration of $240 \mathrm{ig} \mathrm{ml}^{-1}$. The $\mathrm{pH}$ was adjusted between 6.5 and 7 with $1 \mathrm{M} \mathrm{HCl}$. Next a $100 \mathrm{mg} \mathrm{ml}^{-1}$ solution of $N$-(3-Dimethylaminopropyl)$\mathrm{N}$-ethylcarbodiimide hydrochloride (EDAC) was freshly prepared in deionized water and used immediately. To the reaction, $46 \mu \mathrm{l}$ of the EDAC solution (24imol, $15 \mathrm{eq}$ ) was added and the reaction was incubated in a bath sonicator for $2 \mathrm{~h}$, at $5^{\circ} \mathrm{C}$ in the dark. A second coupling was performed with a second aliquot of $24 \mathrm{mg}$ of $\alpha, \omega$-diamino(PEG), $\mathrm{pH}$ adjustment between 6.5 and 7 , and another $46 \mu \mathrm{l}$ of a second freshly prepared $100 \mathrm{mg} \mathrm{ml}^{-1} \mathrm{EDAC}$ solution. The nanoparticles were purified by ultracentrifugation using $10 \mathrm{kDa}$ cutoff centrifugal filters (Millipore Amicon, Billerica, MA) by washing $5 \times$ with deionized water until the filtrate was absent of soluble amine by the ninhydrin test. The particles were characterized by particle size analysis and zeta potential using a Zetasizer Nano S (Malvern Instruments, Westborough, MA). Zeta potential was measured at various $\mathrm{pH}$ values and calculated using the Hückel approximation (Supplementary Figure 2).

Quantification of the diffusion of HIV-1 and beads in CM. WT and $\Delta$ Env viral particles were combined at a $1: 1$ ratio by $\mathrm{p} 24$ content, then PEGylated beads $(200 \mathrm{~nm})$ were added to the mixture based on number of particles per field of view. The virus/bead mixture was then added to mucus at $10 \% \mathrm{v} / \mathrm{v}$ on a slide within a single well of a $0.1-\mathrm{mm} 8$-well imaging spacer (Grace Biolabs, Bend, OR). The virus/bead mixture and mucus were gently mixed using a positive displacement pipette, and a coverslip was used to seal the wells for imaging. Imaging was performed using an EMCCD camera (Cascade II: 512, Photometrics, Tucson, AZ) mounted on an inverted Deltavision microscope (Applied Precision, Issaquah, WA) equipped with a $\times 100$ oil-immersion objective (numerical aperture 1.4) and an environmental chamber kept at $37^{\circ} \mathrm{C}$.

Movies were captured with Softworx Imaging Software (Applied Precision) at a temporal resolution of $150 \mathrm{~ms}$ for a minimum of $60 \mathrm{~s}$. At least 100 particles of each type were imaged in each donor. The virions and beads were tracked in two dimension using IDL software (Exelis Visual Information Solutions, Boulder, CO) for multiple-particle tracking macros. ${ }^{35}$ The time lag-dependent, ensemble- and time-averaged MSD was then calculated in the standard manner. ${ }^{11}$

Statistics. Statistical analyses were performed with Prism 5.0. for Mac OS X (GraphPad Software, La Jolla, CA).

SUPPLEMENTARY MATERIAL is linked to the online version of the paper at http://www.nature.com/mi 


\section{ACKNOWLEDGMENTS}

This work was supported in part by NIH grants to S.A.S./S.A.A (T32AI060523), and T.J.H. (R21Al094584, P01Al082971), and by a Collaboration for AIDS Vaccine Discovery grant to T.J.H. from the Bill and Melinda Gates Foundation (OPP1031734, 38643). We thank Brianne Condron, RN, and Maria Jimenez for coordinating the collection of CM specimens, along with the physicians in the Obstetrics and Gynecology Department at Northwestern Memorial Hospital for obtaining the CM samples. We also thank Angela Fought (Department of Preventive Medicine, Feinberg School of Medicine) for helpful discussions and assistance with interpreting statistical data, and Susan Schader, $\mathrm{PhD}$, for critical review of the manuscript. We would also like to thank the James B Pendleton Charitable Trust for all kind donations towards the work presented here.

\section{DISCLOSURE}

The authors declared no conflict of interest.

(c) 2013 Society for Mucosal Immunology

\section{REFERENCES}

1. Coombs, R.W., Reichelderfer, P.S. \& Landay, A.L. Recent observations on HIV type-1 infection in the genital tract of men and women. AIDS 17, 455-480 (2003).

2. Shattock, R.J., Haynes, B.F., Pulendran, B., Flores, J. \& Esparza, J. Improving defences at the portal of HIV entry: mucosal and innate immunity. PLoS Med. 5, e81 (2008).

3. Linden, S., Sutton, P., Karlsson, N.G., Korolik, V. \& McGuckin, M.A. Mucins in the mucosal barrier to infection. Nat. Mucosal Immunol. 1, 14 (2008).

4. Kutteh, W.H. \& Franklin, R.D. Quantification of immunoglobulins and cytokines in human cervical mucus during each trimester of pregnancy. Am. J. Obstet. Gynecol. 184, 865-872 (2001).

5. Nag, P. et al. Women with cervicovaginal antibody-dependent cellmediated cytotoxicity have lower genital HIV-1 RNA loads. J. Infect. Dis. 190, 1970-1978 (2004).

6. Audie, J.P. et al. Mucin gene expression in the human endocervix. Hum. Reprod. 10, 98-102 (1995).

7. Gipson, I.K. Mucins of the human endocervix. Front. Biosci. 6, D1245-D1255 (2001).

8. Andersch-Bjorkman, Y., Thomsson, K.A., Holmen Larsson, J.M., Ekerhovd, E. \& Hansson, G.C. Large scale identification of proteins, mucins, and their O-glycosylation in the endocervical mucus during the menstrual cycle. Mol. Cell. Proteomics 6, 708-716 (2007).

9. Henderson, M.H. et al. Optical imaging and analysis of human vaginal coating by drug delivery gels. Contraception 75, 142-151 (2007).

10. Huggins, G.R. \& Preti, G. Vaginal odors and secretions. Clin. Obstet. Gynecol. 24, 355-377 (1981).

11. Boukari, H. et al. Movements of HIV-virions in human cervical mucus. Biomacromolecules 10, 2482-2488 (2009).

12. Gipson, I.K. et al. The amount of MUC5B mucin in cervical mucus peaks at midcycle. J. Clin. Endocrinol. Metab. 86, 594-600 (2001).

13. Katz, D.F., Slade, D.A. \& Nakajima, S.T. Analysis of pre-ovulatory changes in cervical mucus hydration and sperm penetrability. Adv. Contracept. 13, 143-151 (1997).

14. Wolf, D.P., Blasco, L., Khan, M.A. \& Litt, M. Human cervical mucus. IV. Viscoelasticity and sperm penetrability during the ovulatory menstrual cycle. Fertil. Steril. 30, 163-169 (1978).

15. Zhu, P. et al. Electron tomography analysis of envelope glycoprotein trimers on HIV and simian immunodeficiency virus virions. Proc. Natl Acad. Sci. USA 100, 15812-15817 (2003).
16. Owen, D.H. \& Katz, D.F. A review of the physical and chemical properties of human semen and the formulation of a semen simulant. J. Androl. 26, 459-469 (2005).

17. Lai, S.K. et al. Rapid transport of large polymeric nanoparticles in fresh undiluted human mucus. Proc. Natl Acad. Sci. USA 104, 1482-1487 (2007).

18. Esser, M.T. et al. Differential incorporation of CD45, CD80 (B7-1), CD86 (B7-2), and major histocompatibility complex class I and II molecules into human immunodeficiency virus type 1 virions and microvesicles: implications for viral pathogenesis and immune regulation. J. Virol. 75, 6173-6182 (2001).

19. Cantin, R., Fortin, J.F. \& Tremblay, M. The amount of host HLA-DR proteins acquired by HIV-1 is virus strain- and cell type-specific. Virology 218, 372-381 (1996).

20. Kolegraff, K., Bostik, P. \& Ansari, A.A. Characterization and role of lentivirus-associated host proteins. Exp. Biol. Med. (Maywood) 231, 252-263 (2006).

21. Boucher, R.C. Evidence for airway surface dehydration as the initiating event in CF airway disease. J. Int. Med. 261, 5-16 (2007).

22. Livraghi, A., Mall, M., Paradiso, A.M., Boucher, R.C. \& Ribeiro, C.M. Modelling dysregulated $\mathrm{Na}+$ absorption in airway epithelial cells with mucosal nystatin treatment. Am. J. Respir. Cell. Mol. Biol. 38, 423-434 (2008).

23. Perez-Vilar, J., Randell, S.H. \& Boucher, R.C. C-Mannosylation of MUC5AC and MUC5B Cys subdomains. Glycobiology 14, 325-337 (2004).

24. Yurewicz, E.C., Matsuura, F. \& Moghissi, K.S. Structural characterization of neutral oligosaccharides of human midcycle cervical mucin. J. Biol. Chem. 257, 2314-2322 (1982).

25. Perelson, A.S., Neumann, A.U., Markowitz, M., Leonard, J.M. \& Ho, D.D. HIV-1 dynamics in vivo: virion clearance rate, infected cell life-span, and viral generation time. Science 271, 1582-1586 (1996).

26. Cole, A.M. \& Cole, A.L. Antimicrobial polypeptides are key anti-HIV-1 effector molecules of cervicovaginal host defense. Am. J. Reprod. Immunol. 59, 27-34 (2008).

27. Sun, L. et al. Human beta-defensins suppress human immunodeficiency virus infection: potential role in mucosal protection. J. Virol. 79, 14318-14329 (2005).

28. Zegels, G., Van Raemdonck, G.A., Coen, E.P., Tjalma, W.A. \& Van Ostade, X.W. Comprehensive proteomic analysis of human cervicalvaginal fluid using colposcopy samples. Proteome Sci. 7, 17 (2009).

29. Owen, D.H. et al. Biophysical analysis of prototype microbicidal gels. J. Pharm. Sci. 96, 661-669 (2007).

30. Kieweg, S.L. \& Katz, D.F. Squeezing flows of vaginal gel formulations relevant to microbicide drug delivery. J. Biomech. Eng. 128, 540-553 (2006).

31. Laga, M. et al. Non-ulcerative sexually transmitted diseases as risk factors for HIV-1 transmission in women: results from a cohort study. AIDS 7, 95-102 (1993)

32. Atashili, J., Poole, C., Ndumbe, P.M., Adimora, A.A. \& Smith, J.S. Bacterial vaginosis and $\mathrm{HIV}$ acquisition: a meta-analysis of published studies. AIDS 22, 1493-1501 (2008).

33. Olmsted, S.S., Meyn, L.A., Rohan, L.C. \& Hillier, S.L. Glycosidase and proteinase activity of anaerobic gram-negative bacteria isolated from women with bacterial vaginosis. Sex Transm. Dis. 30, 257-261 (2003).

34. Boskey, E.R., Moench, T.R., Hees, P.S. \& Cone, R.A. A self-sampling method to obtain large volumes of undiluted cervicovaginal secretions. Sex Transm. Dis. 30, 107-109 (2003).

35. Crocker, J.C. \& Grier, D.G. Methods of digital video microscopy for colloidal studies. J. Colloid Interface Sci. 179, 298-310 (1996). 